\title{
Analyzing on post-impact equivalent velocity of the vehicle side-slipping and rotating
}

\author{
Ma Dan, Yongheng Yue \\ Traffic College, Northeast Forestry University, Harbin 150040, China \\ ayueyyh@126.com
}

\begin{abstract}
Keywords: equivalent adhesion coefficient, road adhesion coefficient, side-sliding and rotation, equivalent velocity.

Abstract. pre-impact speed of the vehicle in the road traffic accident is the important index of dealing with traffic accidents responsibility. We investigate the relation between the road adhesion coefficient and the vehicle side-sliding angle, to constructing adhesion coefficient ellipse equation and then building the precise mathematical model, application on integral principle restructure solving post-impact equivalent velocity mathematical model building. In terms of actual case comparative analysis, the proposed method of in this paper improves $8 \%$ for calculation post-impact velocity of the vehicle than the traditional method in the traffic accident. Therefore the calculated method is great significance for guarantee judicial fairness.
\end{abstract}

\section{Introduction}

Estimated pre-impact velocity of the vehicle in the road traffic accident is the most importance project for judicial identical work ${ }^{[1]}$. As is well known. traffic accident calculation method be difficulty criterion. Obviously, the methods of debating how to deal with some issue about calculating impact speed are directly significant.

In terms of classical mechanics, we can tell that the object with kinetic energy move some stopping places, whose kinetic energy is wholly consumed by the friction working. This kinetic process is described as follows $v=(2 g \varphi S)^{0.5}$, Where $g=9.8 \mathrm{~m} / \mathrm{s}^{2}$ is gravity acceleration; $\varphi$ is friction coefficient mapping; $S$ is sliding distant.

The initial speed of vehicles beginning sliding is solved by $v=(2 g \varphi S)^{0.5[2-4]}$. But on a different impact position and impact angle, with rotation in the process of the vehicle sliding, the road adhesion coefficient is change from them.

\section{Equivalent adhesion coefficient}

The adhesion coefficient is importance for calcultaiting pre-impace speed. The adhesion coefficient is also related with the road material and rough surface and so on ${ }^{[2,3]}$. The concept of the equivalent adhesion coefficient is significant in traffic accident mechanics. Since each tire adhesion coefficient is different, but their integration effect is substituted one parameter that is so called the equivalent adhesion coefficient. This parameter is very convenient analysis and study on issue.

The purpose of this paper does not investigate intrinsic reason on the road adhesion coefficient ${ }^{[3]}$. we only investigate any side-sliding angles how to solve equivalent adhesion coefficient, whose problem is nearly investigated in related literature ${ }^{[4]}$.

$\varphi$ is the road adhesion coefficient, $\varphi_{h}=0.97 \varphi+0.08^{[4,5]}$. Supposed $\vartheta_{\text {is }}$ the vehicle side-sliding angle, then the vehicle motive state is described as follows: 


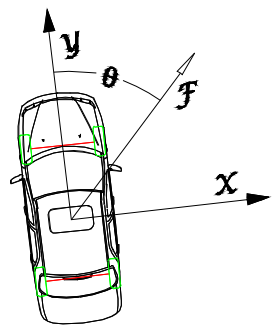

Fig.1 post-impact vehicle side-sliding movement state

$$
\begin{aligned}
& F_{y}=F \cos \vartheta, F_{y}=\varphi_{y} m g \\
& F_{x}=F \sin \vartheta, F_{x}=\varphi_{x} m g
\end{aligned}
$$

If $\vartheta=0^{\circ}$ then $F=m g \varphi ; \vartheta=90^{\circ}$ then $F=m g \varphi_{h}$, according to equation (1) and equation (2) can deduce as:

$$
\frac{\varphi_{y}^{2}}{\varphi^{2}}+\frac{\varphi_{x}^{2}}{(0.97 \varphi+0.08)^{2}}=1
$$

Where $\varphi_{y}=\cos \vartheta, \varphi_{x}=(0.97 \varphi+0.08) \sin \vartheta$. Equation (3) is famous adhesion coefficient ellipse. If $\varphi=0.7$, adhesion coefficient ellipse can be described as follows ${ }^{[6,7]}$ :

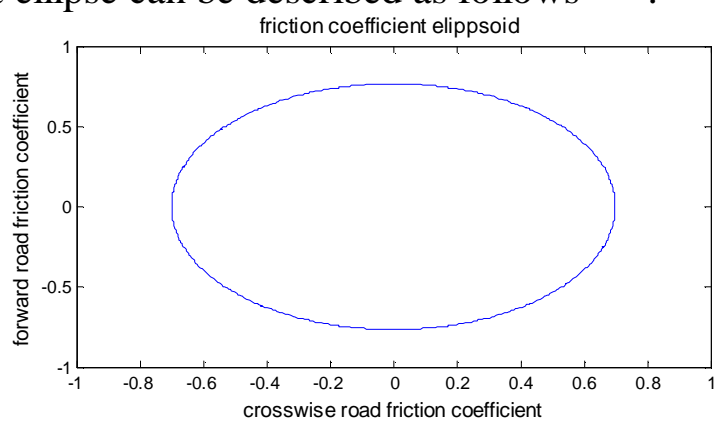

Fig.2 adhesion coefficient ellipse equation

In terms of Fig.2, the equivalent adhesion coefficient is related with side-sliding angle. So equivalent adhesion coefficient is concept as follows:

$$
\varphi_{e}(\vartheta)=\left(\varphi_{y}^{2}+\varphi_{x}^{2}\right)^{0.5}
$$

So we can know that equivalent adhesion coefficient is related with side-sliding angles.

\section{Post-impact equivalent speed}

When the traffic accident occurs, vehicles maybe slide or rotate. So there exists the angle between the orient of the vehicle running and the vehicle mass center trajectory, where angular rotation and angular velocity are variable, but angular acceleration is constant value. After the vehicle has impacted, the motive state of vehicle side-sliding and rotating is described as follows: 


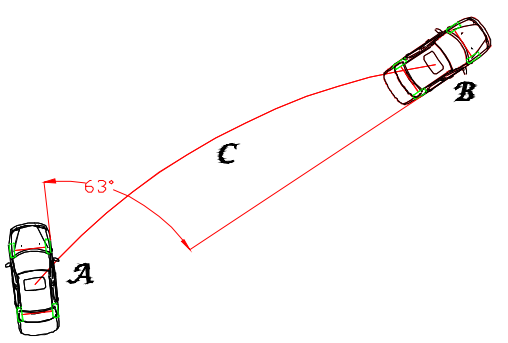

Fig.3 the vehicle post-impact kinetic state

According to tires stamp characteristic on the ground, we ought to analyze post-impact initial state and final state to make sure rotation angle of the accident vehicle.

The vehicle mass center trajectory moves along with curve "ABC" from " $\mathrm{A}$ " position to " $\mathrm{B}$ " position. Because the angle between the vehicle mass center trajectory and initial movement orient is variable in any time. When the post-impact vehicle rotates movement from initial state to final state, because of the vertical of rotation movement orientation and vehicle running trend, so crosswise adhesion coefficient is invariable $\varphi_{x}=(0.97 \varphi+0.08) \sin \vartheta$.

The post-impact vehicle equivalent velocity in Fig. 3 is described as follows:

$$
v_{e}=\left[\sum_{i=1}^{n} 2 g \Omega\left(x_{i}\right) \sigma+\frac{2 g L \alpha \pi}{180}(0.98 \varphi+0.08)\right]^{0.5}
$$

Where

$$
\Omega(x)=\left[\varphi^{2}+(0.08+1.97 \varphi)(0.08-0.03 \varphi) \sin ^{2}\left(\vartheta(0)-\arctan \frac{\partial f(\cdot)}{\partial x}\right)\right]^{0.5} \times\left(1+\left(\frac{\partial f(\cdot)}{\partial x}\right)^{2}\right)_{;}^{0.5} \sigma=\frac{\left|x_{\max }-x_{\min }\right|}{n} .
$$

Where $g$ is gravity acceleration; $\varphi$ is longitudinal adhesion coefficient; $L$ is auto wheel-base; $\alpha_{\text {is }}$ vehicles rotation angle; $f(\cdot)$ is polynomial fitting function of vehicle mass center movement trajectory curve. On the other words, in the Fig.4 curve equation of the vehicle mass center trajectory movement is described as follows: $y=f\left(x^{n}, x^{n-1}, \cdots, x\right) ; x \in R^{+}$. Where the post-impact vehicle mass center movement trajectory is polynomial fitting function, $n_{\text {is proper power. }}$

\section{Vehicles sliding consumption energy}

According to sliding angle concept, the angle between velocity orientation of any position in the curve equation and vehicles initial sliding state is

$$
\vartheta(x)=\vartheta(0)-\arctan \frac{\partial f(\cdot)}{\partial x}
$$

In terms of equation (5), equivalent adhesion coefficient can be described as follows:

$$
\varphi_{e}(x)=\left[\varphi^{2}+(0.08+1.97 \varphi)(0.08-0.03 \varphi) \sin ^{2}\left(\vartheta(0)-\arctan \frac{\partial f(\cdot)}{\partial x}\right)\right]^{0.5}
$$

Since vehicles sliding movement, the consumption energy is described as follows from initial state of the vehicle movement to final state:

$$
E_{1}=\int_{X \in B} m g \varphi_{e}(x) d l=\int_{x_{\min }}^{x_{\max }} m g \varphi_{e}(x)\left(1+\left(\frac{\partial f(\cdot)}{\partial x}\right)^{2}\right)^{0.5} d x=\sum_{i=1}^{n} m g \Omega\left(x_{i}\right) \sigma
$$


Where

$$
\Omega(x)=\left[\varphi^{2}+(0.08+1.97 \varphi)(0.08-0.03 \varphi) \sin ^{2}\left(\vartheta(0)-\arctan \frac{\partial f(\cdot)}{\partial x}\right)\right]^{0.5} \times\left(1+\left(\frac{\partial f(\cdot)}{\partial x}\right)^{2}\right)^{0.5} ; \sigma=\frac{\left|x_{\max }-x_{\min }\right|}{n} .
$$

\section{Vehicles rotation consumption energy}

Supposed vehicles mass center lies in the vehicle geometric center, then vehicles rotation consumption energy is

$$
E_{2}=m g \frac{L \alpha \pi}{180}(0.98 \varphi+0.08)
$$

Where $m$ is an accident vehicle mass, $g$ is gravity acceleration, $\varphi$ is longitudinal adhesion coefficient, $\mathrm{L}$ is vehicles wheelbase, $\alpha$ is vehicles rotation angle.

\section{Vehicles post-impact equivalent velocity}

According to vehicles sliding and rotation consumption energy to calculate post-impact equivalent velocity, in terms of equation (9) and (10) can obtain

$$
v_{e}=\left[\sum_{i=1}^{n} 2 g \Omega\left(x_{i}\right) \sigma+\frac{2 g L \alpha \pi}{180}(0.98 \varphi+0.08)\right]^{0.5}
$$

Where

$$
\Omega(x)=\left[\varphi^{2}+(0.08+1.97 \varphi)(0.08-0.03 \varphi) \sin ^{2}\left(\vartheta(0)-\arctan \frac{\partial f(\cdot)}{\partial x}\right)\right]^{0.5} \times\left(1+\left(\frac{\partial f(\cdot)}{\partial x}\right)^{2}\right)^{0.5} ; \sigma=\frac{\left|x_{\max }-x_{\min }\right|}{n} .
$$

Where $g$ is gravity acceleration; $\varphi$ is longitudinal adhesion coefficient; $L$ is auto wheel-base; $\alpha_{\text {is }}$ vehicles rotation angle; $f(\cdot)$ is polynomial fitting function of vehicle mass center movement trajectory curve.

\section{Analyzing on actual case}

Accident scene being described as follows figure. now in terms of the police investigation data at the scene is to analyze post-impact equivalent velocity of accident vehicles.

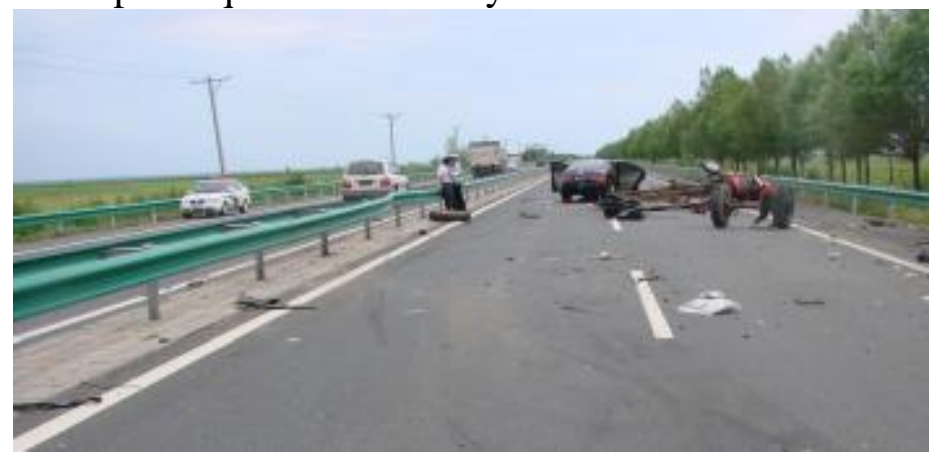

Fig. 5 the road traffic accident scene

According to tires stamp characteristic on the road after collision, Polynomial fitting functions for tire arc marks of vehicles sliding and rotation obtains curve equation by excel software as follows: 


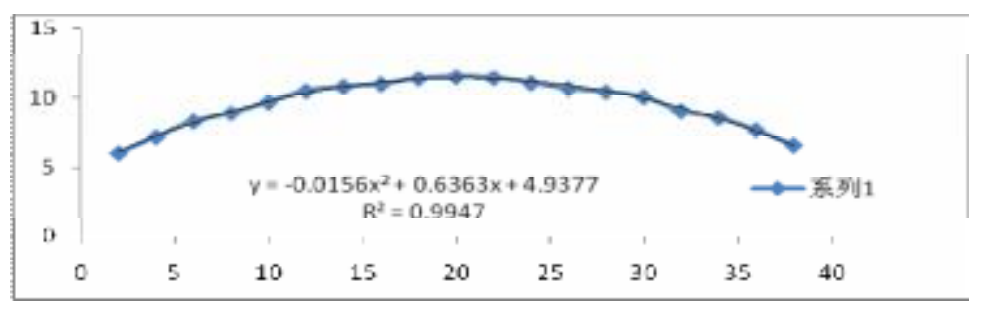

for $\quad y=-0.0156 x^{2}+0.6363 x+4.9377, \vartheta=\arctan \frac{d y}{d x}=-0.0312 x+0.6363 \quad$ So, $\quad \vartheta(x=0)=32.47^{\circ}$ and $\vartheta(x=38)=-28.78^{\circ}$, accident vehicle rotation angle is $\vartheta=61.25^{\circ}$.

\section{Calculation rotation equivalent velocity}

$$
v_{R}=\left[\frac{2 g L \vartheta \pi}{180}(0.98 \varphi+0.08)\right]^{0.5}=\left[\frac{2 \times 9.8 \times 2.65 \times 61.25 \times 3.14}{180}(0.98 \times 0.7+0.08)\right]^{0.5}=6.52 \mathrm{~m} / \mathrm{s}
$$

\section{Calculation sliding equivalent velocity}

Let $x_{\min }=0, x_{\max }=38 m, n=100$,step length is $0.38 m$ :

$$
v_{s}=\left(2 g \sum_{i=1}^{n}\left[\varphi^{2}+(0.08+1.97 \varphi)(0.08-0.03 \varphi) \sin ^{2}\left(\vartheta(0)-\arctan \frac{\partial f(\cdot)}{\partial x}\right)\right]^{0.5} \times\left(1+\left(\frac{\partial f(\cdot)}{\partial x}\right)^{2}\right)^{0.5} \frac{\left|x_{\max }-x_{\min }\right|}{n}\right)^{0.5}=23.9 \mathrm{~m} / \mathrm{s}
$$

o post-impact equivalent velocity is $v_{e}=89 \mathrm{~km} / \mathrm{h}$; If application tradition method calculation post-impact velocity is $v=82 \mathrm{~km} / \mathrm{h}$. For this case, the method of this paper improves $8 \%$ calculation accuracy, so this method is significant for calculating accident vehicles pre-impact velocity.

\section{Conclusions}

The analysis of pre-impact velocity of accident vehicles is mostly important technical index in the course analysis of traffic accident with sliding and rotation movement of post-impact vehicles. When vehicles sliding, the road adhesion coefficient is anisotropic, namely sliding adhesion coefficient is not longitudinal adhesion coefficient, but crosswise adhesion coefficient. According to this point we build accurate mathematical model to describe this physical process, improving calculation accuracy of post-impact equivalent velocity, further more providing the scientific and technical basis for analysis of pre-impact velocity of accident vehicles.

\section{Reference}

[1] Dario Vangi. Simplified method for evaluating energy loss in vehicle collisions. Accident Analysis and Prevention. 2009,36:633-641.

[2] Jun Xu, Yibing Li, Guangquan Lu, Wei Zhou. Reconstruction model of vehicle impact speed in pedestrian-vehicle accident. 2009,36:783-788.

[3] Bradley E., Boyd D., James J., Gunter P. Vehicle speed affects both pre-skid braking kinematics and average tire roadway friction. Accident Analysis and Prevention, 2004, 36:829-840.

[4] Goudie, D.W., Bowler, J.J., Brown, C.A., Heinrichs, B.E., Siegmund,G.P. Tire friction during locked wheel braking.In: Accident Reconstruction: Analysis, Simulation, and Visualization (SP-1491). Society of Automotive Engineers, Warren dale, PA, pp. 479-490.

[5] Yongheng Yue, Baoquan, Qingdong Xia. Analysis on no-slip equivalent friction coefficient. 2010 International Conference on Computer, Mechatronics, Control and Electronic Engineering. Wuhan, 2010:322-325.

[6] Lin Yang. Traffic accident assessment [M]. Beijing: People Transport Press, 2001. 
[7] Guo Konghui. Anisotropy adhesion coefficient under different condition on tires mechanics character's Unified Theory Model [J]. China mechanical engineering, 1996, 7(4):90-93. 